Review

\title{
Echocardiography in Infective Endocarditis: An Update on its Usefulness: How, When, How Much and Where?
}

\author{
Josep Maria Frigola, Sergio Moral, Pau Vilardell and Ramon Brugada \\ Department of Cardiology, Hospital Universitari Doctor Josep Trueta, Spain
}

\author{
Article history \\ Received: 01-04-2021 \\ Revised: 10-05-2021 \\ Accepted: 15-05-2021 \\ Corresponding Author: \\ Josep Maria Frigola \\ Department of Cardiology, \\ Hospital Universitari Doctor \\ Josep Trueta, Spain \\ Email: josepm.frigola@ias.cat
}

\begin{abstract}
Echocardiography is the main imaging technique in the diagnosis of Infective Endocarditis (IE). In 85-90\% of cases this diagnosis is based on some echocardiographic criteria. Transthoracic echocardiography is an inexpensive test, quickly accessible without side effects, which has become the initial imaging technique of choice in the diagnosis of IE, as well as in the assessment of complications and follow-up. Transesophageal echocardiography also plays an important role in IE, increasing the diagnostic accuracy to almost $90 \%$, especially in complicated cases. The new technologies implemented in the last decade have improved the assessment of these patients. Among them, 3D echocardiography has proven to be useful in the localization of periprosthetic leaks, valve perforations, fistulae as well as in the extensions of aneurysms and abscesses. The increased availability of echographs and the reduction in their size have led to perform hand-held cardiac ultrasound not only by cardiologists, but also by intesivists, anesthesiologists and internists among other specialties. This review is intended to help the clinician to better understand the usefulness of echocardiographic technique in the IE assessment and to review its new roles with the evolution of technology.
\end{abstract}

Keywords: Echocardiography, Infective, Endocarditis, Diagnosis

\section{Introduction}

Infective Endocarditis (IE) is an infectious cardiac disease with an incidence of 5-10 episodes per 100,000 people/year, being higher in the elderly (Tornos et al., 2011). In those over 80 years old, the incidence is around 37 episodes per 100,000 peoples/year (Shah et al., 2020). It presents a high in-hospital mortality, approximately $20-25 \%$, without improvement in the recent decades despite the evolution of medical and surgical management (Tran et al., 2017; Khan et al., 2020).

The current profile of IE patients has been modified due to social changes in developed countries in recent years. Nowadays, the population has more longevity, more comorbidity and in consequence a more frailty people with higher risk to develop IE (Thuny et al., 2012). Microorganisms have also changed in the last decades (Martín-Dávila et al., 2005). The greater number of prosthetic valves, of intracardiac devices (pacemakers, intracavitary defibrillators...), of hemodialysis patients and risk factors, such as diabetes mellitus, have caused a higher incidence of infections by more aggressive microorganisms like Staphylococcus Aureus, that would explain the non-decrease in mortality from this lethal disease (Pant et al., 2015; García-Granja et al., 2020).

Echocardiography is the main diagnostic tool in IE. Transthoracic Echocardiography (TTE) is an inexpensive test, quickly accessible without side effects, which has become the initial imaging technique of choice in the diagnosis of IE, as well as in the assessment of complications and follow-up (Habib et al., 2010). Transesophageal Echocardiography (TEE) also plays an important role, increasing the diagnostic yield from $50-70 \%$ of TTE to almost $90 \%$ (Fernandez-Hidalgo and Almirante, 2012). In addition, the new technologies implemented in the last decade have improved the assessment of these cases. Among them, 3D echocardiography has proven to be useful in the localization of periprosthetic leaks, valve perforations, fistulae as well as in the extensions of aneurysms and abscesses (Bhattacharyya et al., 2014). Furthermore, the increased availability of echographs and the 
reduction in their size have led to perform hand-held cardiac ultrasound not only by cardiologists, but also by intesivists, anesthesiologists and internists among other specialties.

This review is intended to help the clinician to better understand the usefulness of echocardiographic technique in the IE assessment and to review its new roles with the evolution of technology.

\section{Echocardiography in IE Diagnosis: How to do it?}

Echocardiography has a great importance for the IE diagnosis and also for the severity assessment of the disease, providing prognostic data such as embolic risk and the follow up evaluation of these patients (Afonso et al., 2017). Both TTE and TEE should be performed in a high-suspicious IE patient. TEE allows better evaluation in several situations in which TTE has limited sensitivity such as prosthetic valve IE, small vegetations and in the presence of perivalvular abscess (Erba et al., 2019).

\section{Major and Minor Criteria and the Role of Echocardiography}

IE diagnosis is established by the modified Duke criteria based on clinical, echocardiographic and biomarker findings, as well as microbiological results from blood cultures and serology tests (Li et al., 2000). These criteria showed a sensitivity of $80 \%$. In 2015 , the working group of the European Society of Cardiology (ESC) proposed a modification of these criteria due to technological advances in cardiac imaging tests in order to increase the diagnostic sensitivity of the classical Duke criteria especially in more complex diagnostic cases. In $85-90 \%$ of cases the diagnosis of IE is based on some echocardiographic criteria and only about $10 \%$ of patients are diagnosed without any imaging-reported lesion (Habib et al., 2010; Evangelista and Gonzalez-Alujas, 2004; Sochowski and Chan, 1993) (Table 1).

\section{Echocardiography IE Lesions}

IE produces some anatomical alterations which can be visualized by echocardiographic studies and have significant clinical implications for diagnosis and prognosis. Those usually are divided in three groups (Habib et al., 2010):

- Vegetation: Mass located in the low-pressure chambers of the cardiac cavities at any point of the endocardium, valvular and/or subvalvular apparatus

- Abscess formation: Perivalvular cavity mainly in aortic valve or valvular prosthesis. Usually located at the level of the aortic annulus and mitro-aortic fibrous tissue

- Tissue destruction: Such as valve prolapse and perforation, chordal rupture or valvular aneurysms

The major echocardiographic criteria from the modified Duke criteria by ESC guidelines (Habib et al., 2015) are the following (Table 1 and Fig. 1).

\section{Vegetation}

Intracardiac mass mainly lodged in low-pressure chambers implanted in valves, subvalvular apparatus or elsewhere in the endocardium and in prosthetic material (intracardiac devices such as pacemakers, defibrillators, septal defect closure devices, atrial appendage closure devices...) (Fig. 2).

Table 1: Modified duke criteria for IE diagnosis

Major criteria

1. Blood culture positive for IE:

a. Typical microorganisms consistent with IE from two separate blood cultures: Streptococcus viridans, S. gallolyticus (S. bovis), HACEK group, Staphylococcus aureus or community -acquired enterococci, in the absence of a primary focus.

$b$. Microorganisms consistent with IE from persistently positive blood cultures, defined as follows: At least two positive blood cultures of blood samples drawn $>12 \mathrm{~h}$ apart; or all the three or a majority of 4 separate cultures of blood (with first and last sample drawn at least $1 \mathrm{~h}$ apart).

c. Single positive blood culture for Coxiella burnettii or antiphase I IgG antibody titer $>1: 800$

2. Positive cardiac imaging finding for IE:

a. Echocardiography positive for IE (TEE recommended in patients with prosthetic valves, rated at least "possible IE" by clinical criteria, or complicated IE (paravalvular abscess); TTE as first test in other patients), defined as follows: Oscillating intracardiac mass on valve or supporting structures, in the path of regurgitant jet/Abscess/New partial dehiscence of prosthetic valve. New valvular regurgitation (worsening or chan ging).

$b$. Abnormal activity around the prosthetic valve implant site detected by 18F-FDG PET/CT (only if the prosthesis has been implanted for more than 3 months) or SPECT/CT with isotope-labeled leukocytes.

c. Paravalvular lesions in CT scan.

1. Predispositions such as predisposing cardiac disease or injecting drug use.

2. Fever $>38^{\circ} \mathrm{C}$

3. Vascular phenomena (including those detected only by imaging): major arterial emboli, septic pulmonary infarcts, infectious (mycotic) aneurysm, intracranial hemorrhage, conjunctival hemorrhages and Janeway lesions.

4. Immune phenomena: glomerulonephritis, Osler nodules, Roth's spots and rheumatoid factor.

5. Microbiological evidence: Positive blood culture that does not meet a major criterion listed or serological evidence of active infection with a microorganism compatible with IE. 


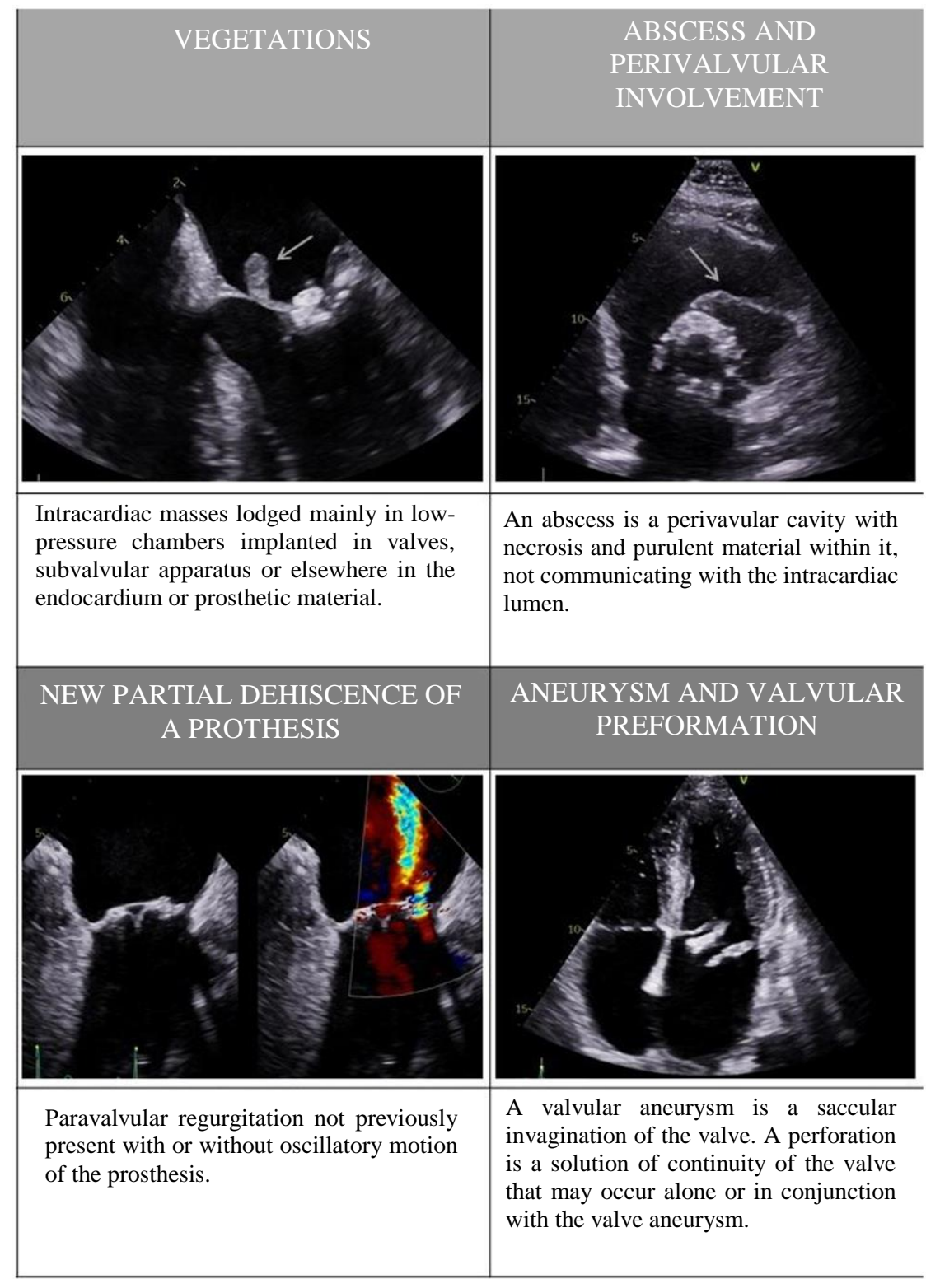

Fig. 1: Different echocardiographic images showing the most frequent complications found in imaging studies in infective endocarditis

Video 1: https://thescipub.com/video/ajidsp.2021.76.87/1.mp4

Video 2: https://thescipub.com/video/ajidsp.2021.76.87/2.mp4

Video 3: https://thescipub.com/video/ajidsp.2021.76.87/3.mp4

Video 4: https://thescipub.com/video/ajidsp.2021.76.87/4.mp4

The typical vegetation is a vibrated mass with oscillating motion attached to a cardiac valve lodged in the low-pressure chambers (atrium in atrioventricular valves and in the outflow tract of the left or right ventricle in the aortic and pulmonary valve respectively). However, a vegetation can also be a fixed adherent mass in other atypical locations. TTE show a sensitivity of 50-70\% for the diagnosis of vegetations, but it should be taken into account that a suboptimal ultrasound window and small vegetations would significantly decrease this diagnostic sensitivity. TEE is especially useful in these cases for the detection of vegetations, increasing sensitivity up to $90 \%$ (MacKay et al., 2020). TEE has also demonstrated to be clearly superior in the IE diagnosis over valvular prostheses, intracavitary devices and pacemakers. The reverberations, artifacts and acoustic shadows produced by devices and prostheses make it difficult to visualize vegetations correctly with TTE, improving sensitivity 
with TEE. Nevertheless, the diagnostic specificity of TTE and TEE vegetations is not $100 \%$. Caution should be exercised in the face of certain findings that can lead to an erroneous diagnosis of bacterial IE, the main ones being: Thrombi, tumors, remnants of previous IE, surgical debris, fibrin filament, non-infectious vegetations in marantic IE, Lambl's excrescences, cusp prolapsed and degenerative changes (Fig. 3 and Table 2) (Mügge et al., 1989).
Table 2: Main pitfalls in the diagnosis of vegetations

\section{-Thrombus.}

-Cardiac tumors: Fibroelastoma, atrial myxoma,...

-Remains of vegetations of previous IE.

-Lambl's excrescence.

-Marantic IE: non-infective IE.

-Valvular degenerative changes.

-Prolapse of valvular cusps.

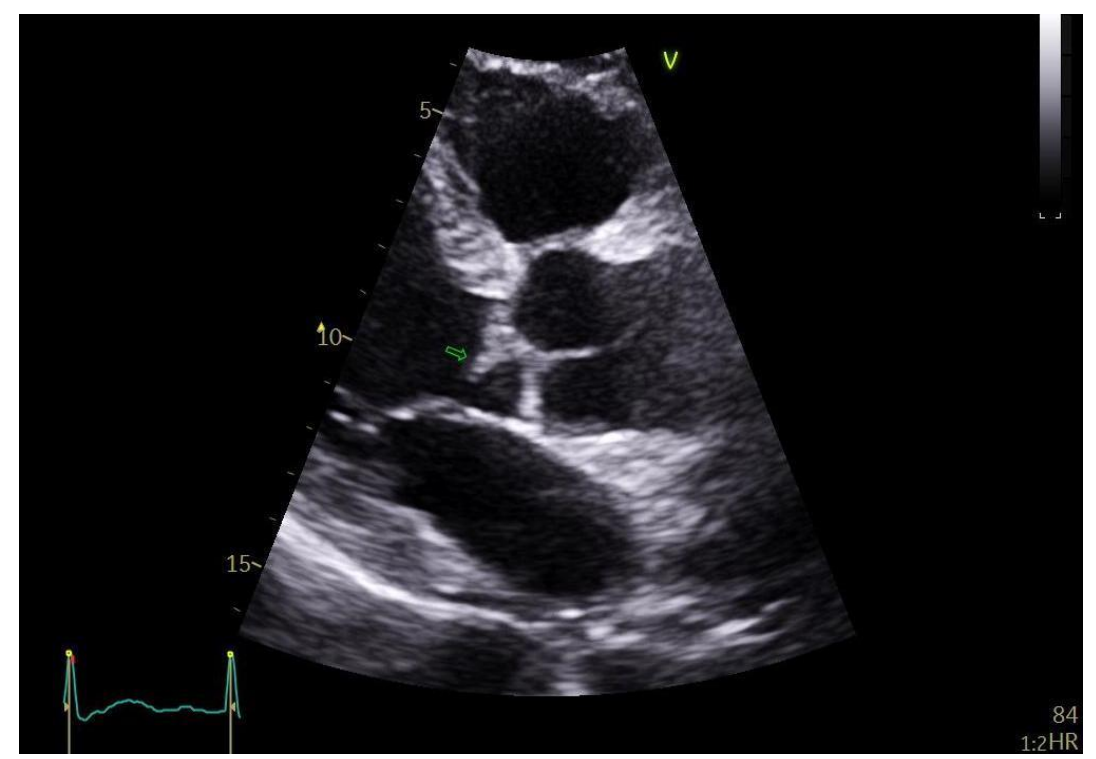

Fig. 2: Transthoracic echocardiography image demonstrating a native aortic IE: The green arrow shows a vegetation attached to the aortic valve

Video 5: https://thescipub.com/video/ajidsp.2021.76.87/5.mp4

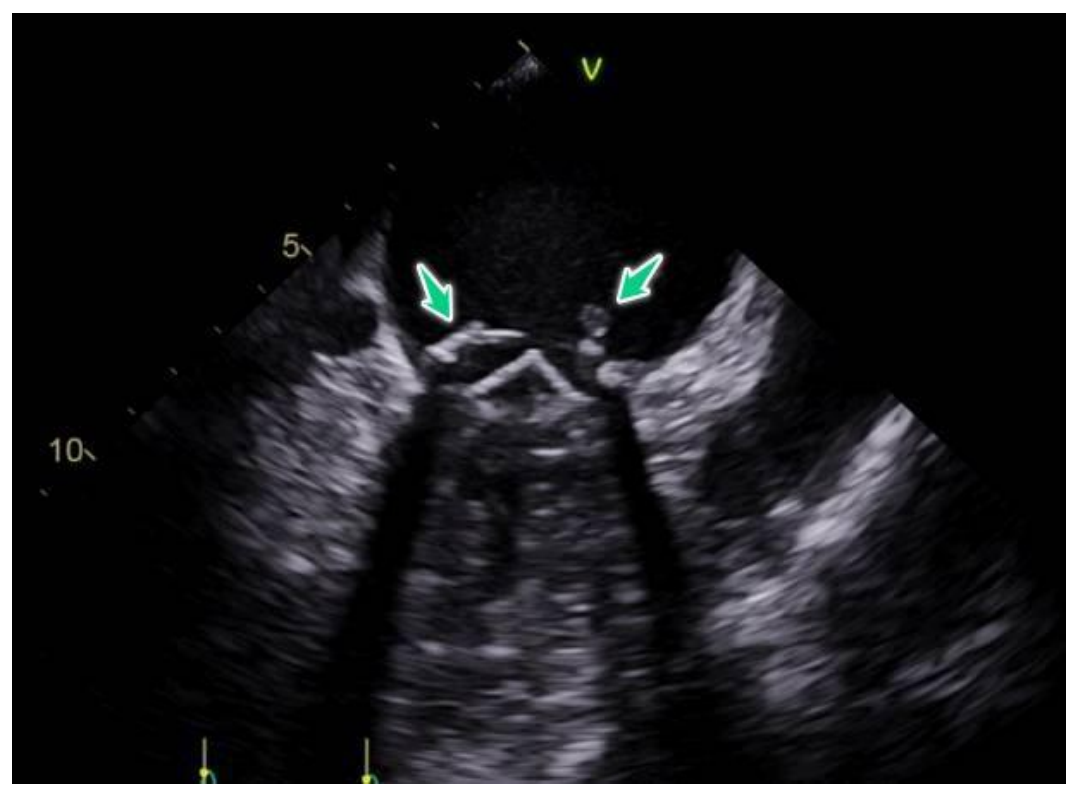

Fig. 3: Transesophageal echocardiography image depicting a mobile mass attached at the level of the annulus of a mechanical mitral prosthesis in a patient admitted for a stroke episode. In this case, the mass is a thrombus adhered to the prosthesis, not a vegetation (green arrows)

Video 6: https://thescipub.com/video/ajidsp.2021.76.87/6.mp4 


\section{Abscess and Perivalvular Involvement}

\section{Abscess}

It is defined as infective necrosis of the valve annulus structure with purulent material inside without communication with the intracardiac lumen. Echocardiographically it is visualized as an echolucent or echodense thickening zone within the myocardium or annular area. The most frequent location is at the aortic valve, in the valvular annulus or mitro-aortic fibrosis. The diagnosis in early stages with small abscesses and with little time of evolution could be difficult. The sensitivity of TTE for its diagnosis is 50\% while it reaches $90 \%$ with TEE. Moreover, both tests (TTE and TEE) showed a high specificity for the diagnosis reaching 90\% (Daniel et al., 1991). Given that abscess is a dynamic process, a new echocardiographic control is recommended when the initial study is inconclusive. In case of aortic prostheses, the anterior zone of the annulus could be better assessed with TTE than with TEE, so both tests are necessary and complementary (Yuan et al., 2019).

\section{Perivalvular Involvement: Pseudoaneurysm and Fistula}

A pseudoaneurysm is an echo-free perivalvular cavity communicating with the intracardiac lumen. Echocardiographically it is depicted as a pulsatile cavity with color Doppler-visualized communication with the cardiac lumen.

In the formation of abscess and perivalvular involvement Computed Tomography (CT) has demonstrated to be superior than echocardiography in the diagnosis of those lesions, with greater accuracy for its detection due to its higher spatial resolution (del Prado Díaz et al., 2016).

Fistulas are another perivalvular IE complication, defined as a communication between two cavities through a perforation. Communication is usually detected by color Doppler. 3D echocardiography is especially useful in perivalvular involvement, providing complementary information to TTE and TEE, since it allows to visualize the structures in multiple planes (Bhattacharyya et al., 2014; Liu et al., 2009).

\section{Aneurysm and Valvular Perforation}

A valvular aneurysm consists of a saccular invagination of the valve. The valvular perforation is a solution of continuity of the valve that may occur alone or accompanied by the valve aneurysm. The most frequent location is usually in the anterior leaflet of the mitral valve originating through the regurgitation jet of an infected aortic valve. Frequently, perforations are usually well visualized with color Doppler in TTE, but the sensitivity of TEE is higher. 3D echocardiography is very useful in these cases for better anatomical assessment and localization of the perforation. This complication usually causes severe valvular regurgitation. For this reason, the left and right ventricular size and function assessment is crucial, as well as the hemodynamic status of the patient (pulmonary pressure, diastolic function...) (Fig. 4).

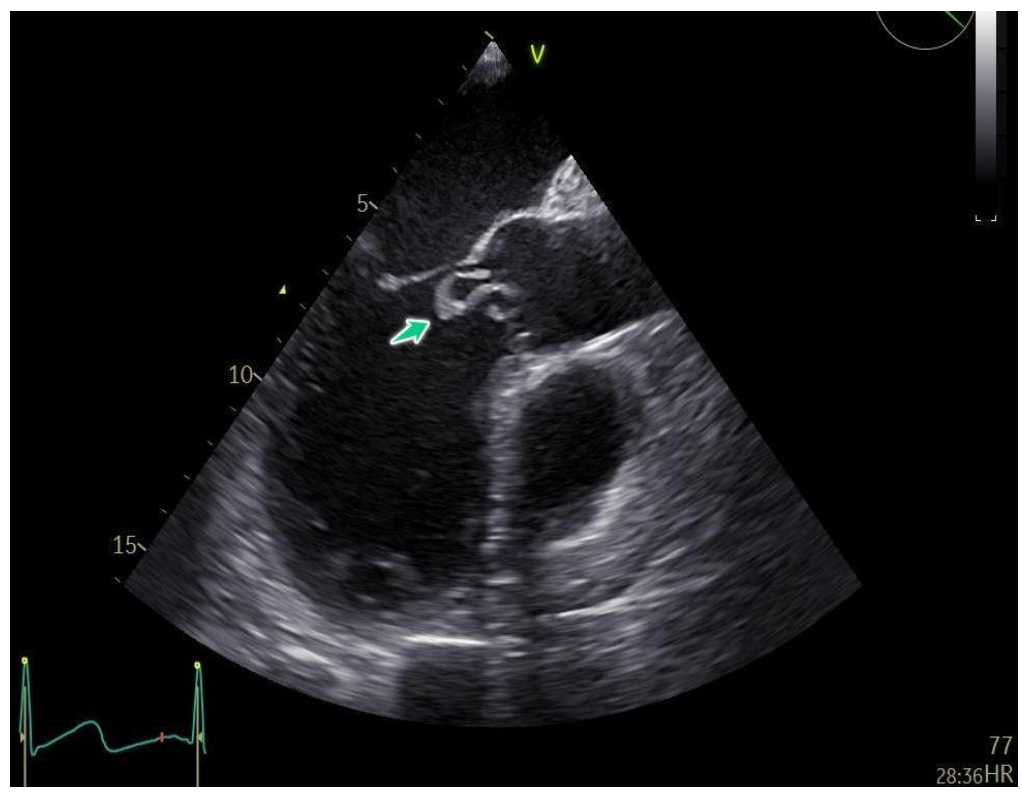

Fig. 4: Transesophageal echocardiography image showing a native aortic IE. Green arrow points to an aneurysm and perforation of the non-coronary leaflet secondary to IE due to Staphylococcus Aureus

Video 7: https://thescipub.com/video/ajidsp.2021.76.87/7.mp4 


\section{New Partial Dehiscence of a Prosthesis}

IE should be suspected in the appearance of a new dehiscence in a prosthesis even in the absence of vegetation or valvular abscess. Echocardiographic study usually visualizes paravalvular regurgitation not previously present with or without oscillatory movement of the prosthesis. In this case, the diagnostic sensitivity of TTE is low, especially in mechanical prostheses at mitral level. The study by TEE and 3D echocardiography will confirm the diagnosis with a sensitivity of $>90 \%$ (Habib et al., 2015).

\section{Echocardiography in IE Diagnosis: When to Perform the Study?}

Echocardiography (TTE or TEE) is the gold standard technique for IE diagnosis and presented an essential role in the management and monitoring of these patients. Both European and American guidelines (ESC, ACC and AHA) recommended TTE for all patients with suspected IE with a recommendation class I (Habib et al., 2015; Otto et al., 2020; Nishimura et al., 2014).

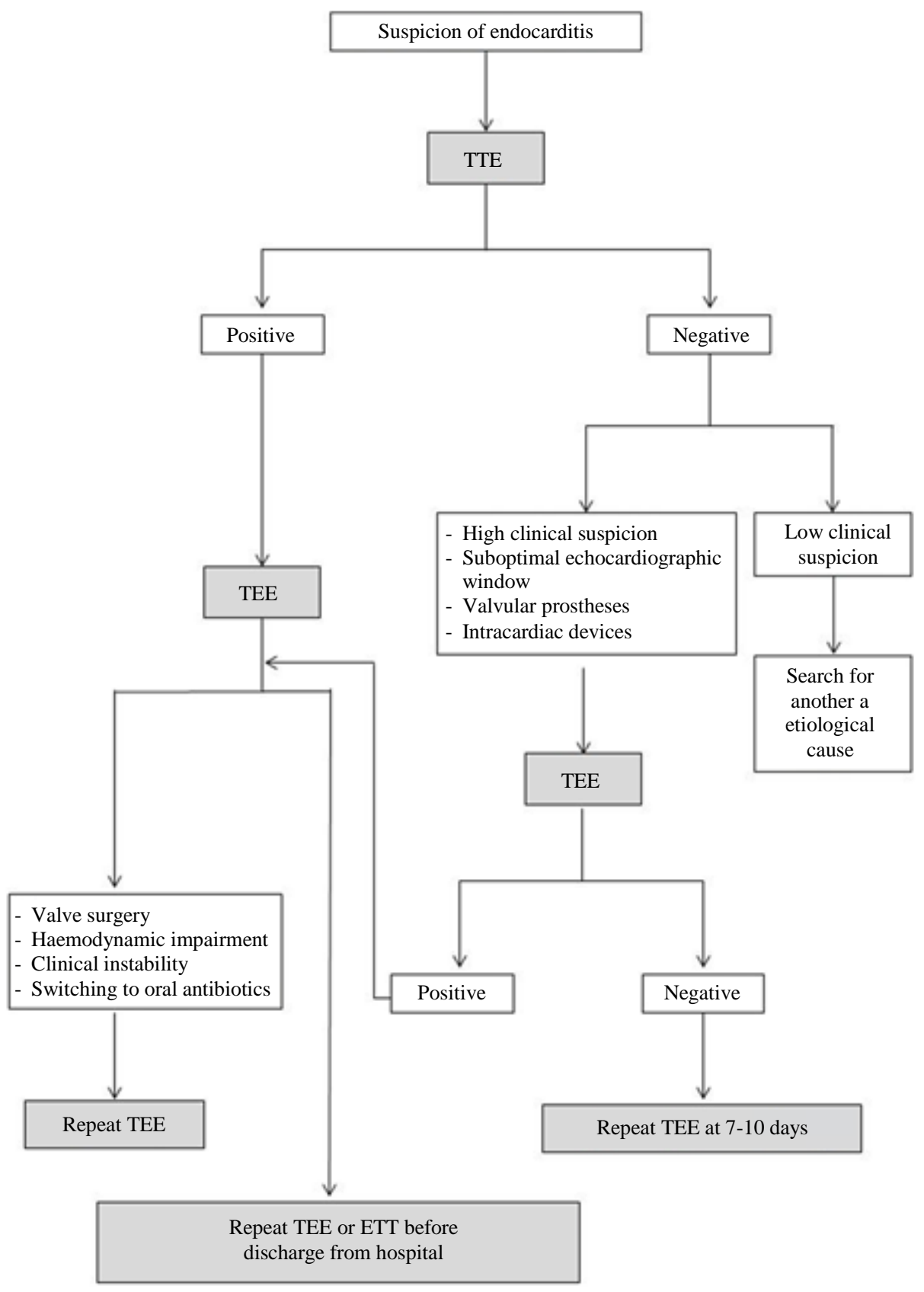

Fig. 5: TTE and TEE algorithm for clinical IE suspicion 
TTE or TEE should also be performed in the presence of clinical or hemodynamic deterioration to rule out valve and/or ventricular dysfunction and hemodynamic status. In all patients with a diagnosis or high suspicion of IE in whom TTE is not diagnostic, TEE is indicated. Common indications include: Suboptimal window, mechanical prostheses, small vegestations, degenerated valves, perivalvular compromise, etc. Likewise, the ACC/AHA guidelines (Nishimura et al., 2014) recommended performing a TEE when IE is suspected in patients with an intracardiac device, Staphylococcus Aureus bacteremia without apparent focus, when antibiotic treatment is changed to the oral management and 3 days before the end of treatment. Intraoperative TEE is also recommended for all patients requiring heart valve surgery for IE. Finally, TEE is recommended for patients with valvular prostheses without bacteremia, but with persistent fever (Fig. 5).

\section{TTE in the Initial Prognostic IE Assessment}

TTE is also the initial imaging technique for assessing the prognosis of those cases. Echocardiographic findings of poor prognosis would be the presence of severe valve dysfunction in native or prosthetic valve, the presence of perivalvular involvement, findings suggesting hemodynamic compromise: Low ejection fraction of the left and right ventricle, severe pulmonary hypertension, early closure of the mitral valve and high risk of embolism (Cabell et al., 2002; Thuny et al., 2005).

\section{Risk of Embolism}

The occurrence of both clinical or silent embolism in IE leads to a high mortality and morbidity rate in both leftsided and right-sided IE (Thuny et al., 2007). The risk is elevated during the first two weeks of antibiotic treatment initiation being between 6-21\% (Habib, 2005). In the prediction of echocardiographic embolic risk, we should assess the size, number and mobility of vegetations, as well as their location. A high risk of embolism is considered for vegetations $>10 \mathrm{~mm}$ in length and it increases even more for those $>15 \mathrm{~mm}$ and mobile. The risk is extreme in those $>30 \mathrm{~mm}$. With regard to location, those located on the mitral valve are of greatest embolic risk. Another parameter to be taken into account is the evolution once antibiotic treatment has been initiated. An increase in the size of vegetations after starting antibiotic therapy is considered a poor prognosis factor with high embolic risk (Fig. 6) (Habib, 2005; Habib et al., 2019).

\section{TTE in IE Follow-Up}

TTE is an essential tool in the initial follow up and control of IE once antibiotic treatment has been iniciated. The frequency will depend on the initial clinical and echocardiographic characteristics. In uncomplicated IE it would be advisable to perform a weekly TTE. A good evolution will show a progressive reduction in the size of the vegetations, as well as a change in their characteristics: Less mobility and increased echogenicity (Habib et al., 2010). Thus, a reduction in size may indicate a good prognosis and a reduction in embolic risk.

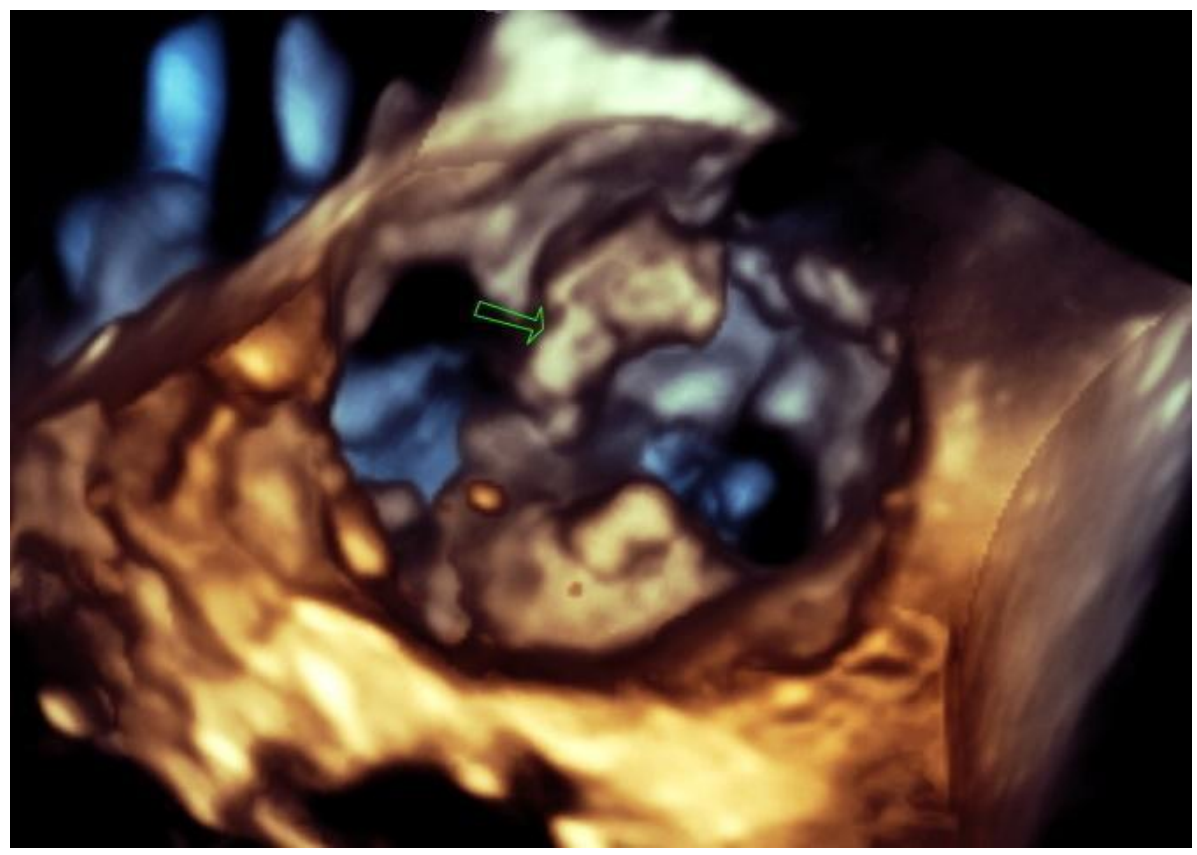

Fig. 6: 3D Transesophageal echocardiography view of mitral valve. A high embolic risk vegetation is detected (green arrow) Video 8: https://thescipub.com/video/ajidsp.2021.76.87/8.mp4 
In the presence of clinical or hemodynamic deterioration it is mandatory to perform a TTE to rule out valvular dysfunction and assessment of the severity of hemodynamic compromise, as well as detection of increased size and number of vegetations indicating therapeutic failure (Vilacosta et al., 2015).

\section{Long-Term Follow-Up}

Before hospital discharge it is necessary to perform a TTE and/or TEE for subsequent comparisons. Given the high recurrence and therapeutic failures during the first months (2-6\%) (Fernandez-Hidalgo and Almirante, 2012; Heiro et al., 2008), it is advisable to perform serial TTE during the first year: At 1, 3, 6 and 12 months after the end of antibiotic treatment.

\section{Echocardiography in IE Diagnosis: How Much do we have to use the Technique?}

In all patients with suspected IE, TTE is recommended to identify vegetations, characterize hemodynamics, assess the severity of valvular lesions, evaluate ventricular function, calculate pulmonary pressure and detect complications (Liu et al., 2009). Normal TTE and TEE do not exclude the diagnosis of IE, since their sensitivity is $70 \%$ for TTE and $90 \%$ for TEE (Habib et al., 2010). If the clinical suspicion is high, it is recommended to repeat the study after 7-10 days, so we can detect changes such as the appearance of complications, growth of vegetations or perivalvular abscesses. Repeating a thrid echocardiography if the second study is negative will not increase the diagnostic sensitivity (Vieira et al., 2004). The specificity of TTE and TEE is about $90 \%$ and we must also take false positives into account (Table 2). However, there are high-risk cases in which prognostic information is of vital importance and determines the treatment to be performed. In these patients, the frequency of repetition of the study should be higher or the period of time to repeat the echocardiography should be shortened, since the risk of presenting the disease or developing complications is higher and the decision must be supported by the Endocarditis Team. The factors to be taken into account are usually divided into cardiovascular and non-cardiovascular risk factors.

\section{Cardiovascular Risk Factors}

Until a few decades ago, rheumatic valve disease and congenital heart disease were the most important predisposing factors for the development of IE. Currently, the most important causes are degenerative valve disease, mainly mitral valve prolapse and cardiac prostheses. Also noteworthy is the significant increase of IE in patients with pacemakers and cardiac devices, which may account for $3 \%$ of all causes (Revilla et al.,
2008; Ortiz et al., 2014). The incidence of IE in patients without apparent heart disease has increased in recent years and they now account for $57 \%$ of cases.

\section{Non-Cardiovascular Risk Factors}

Non-cardiovascular risk factors included elderly, liver cirrhosis, addiction to parenteral drugs and mainly interactions related to health system (hemodialysis, bladder catheters, vascular catheters...) (Ortiz-Bautista et al., 2015; Olmos et al., 2017).

\section{Echocardiography in IE Diagnosis: Where can it be Perfomed?}

Although most cases and diagnoses are performed in the cardiac imaging laboratory (Afonso et al., 2017), the increased availability of echographs and the reduction in their size have led to perform hand-held cardiac ultrasound, also called Point-Of-Care Ultrasound (POCUS), not only by cardiologists, but also by intensivists, anesthesiologists and internists among other specialties.

\section{Intraoperative Echocardiography}

Intraoperative TEE is essential before any cardiac surgery in a patient with IE (AHA/ACC Indication class I) (Nishimura et al., 2014). Pre-pump TEE is also strongly recommended, since the findings in the last echocardiographic control and the patient's conditions may have changed. IE is a dynamic process, vegetations may have grown and there may be an expansion of the process locally with greater tissue destruction. It will help to better plan the subsequent surgery. Post-pump TEE will give us information on the result of the surgery, especially when conservative or very complex surgery is performed (MacKay et al., 2020). In up to $10 \%$ of the operations it can lead to perform a second pump entry and to change the pre-surgery plans in $11 \%$ (Shapira et al., 2007).

\section{Echocardiography in Intensive Care Unit}

IE admission to Intensive Care Unit (ICU) is common. About $50 \%$ of patients will be admitted with an established diagnosis and the remainder will be diagnosed during their ICU stay. Most will be admitted for heart failure, sepsis, neurological deterioration or shock. TTE and TEE are indicated as first-line imaging when there is a clinical suspicion of IE in the ICU. TTE in the ICU has a lower diagnostic sensitivity than that performed in an echocardiography laboratory: Studies are usually performed in intubated patients with mechanical ventilation, with less capacity for mobilization with equipment with lower performance. All these limitations decrease the sensitivity of echocardiography for IE diagnosis to $33 \%$ (Keynan et al., 2013). TEE will be complementary to TTE in the ICU, increasing diagnostic sensitivity to $90 \%$. TEE in the ICU will have added 
difficulties due to patients with critical pathologies, hemodynamic instability and mechanical ventilation. However, its safety remains high with a very low number of complications $(0.5 \%)$ (Hilberath et al., 2010).

\section{Echocardiography in the Emergency Department}

The mobility and relative low cost of echocardiography devices has led to a wide diffusion and use of echocardiography in Emergency Departments (ED). Echocardiography in ED allows a rapid diagnosis at the bedside, shortening the time it takes to perform the study, especially in cases that the patient cannot be transferred to a cardiac imaging unit. Given the high IE mortality, early diagnosis at ED and initiation of antibiotic treatment is crucial for the good evolution of the process (Seif et al., 2013). TTE and POCUS are very useful for positive diagnosis, being less sensitive for the diagnosis of exclusion, especially in small vegetations and right cavities (Fischer and Baduashvili, 2019). In these situations of high clinical suspicion and high probability with a negative TTE or POCUS, the study should be completed as soon as possible with TEE and performed by trained personnel and experts in cardiac imaging in order to minimize possible diagnostic errors.

\section{POCUS in the IE Diagnosis}

POCUS is understood as the limited and directed use of echocardiography as an extension of physical examination by cardiologists and non-cardiologists, with the aim of improving the diagnostic, prognostic and therapeutic assessment provided by conventional physical examination (de Isla et al., 2018). POCUS does not replace a regular TTE study in the echocardiography laboratory. When the POCUS detects abnormal echocardiographic findings that raise the suspicion of heart disease, the study should be completed with a TTE. It would be useful in the evaluation of shock of unknown origin, in the presence of neurological embolic events and in case of suspected heart failure of unclear cause. POCUS could also assess left and right ventricular function, blood volume level, presence of pericardial effusion and cardiac tamponade. POCUS also could detect valvular abnormalities and the presence of cardiac masses or vegetations. These findings should be confirmed by means of a regulated study. Moreover, the fact that these alterations are not detected in POCUS does not rule out these diagnoses. POCUS could help to establish an initial diagnostic suspicion of IE, allowing rapid initiation of treatment and reduction of the morbidity associated with the illness (Taylor et al., 2017; Bugg and Berona, 2016).

\section{Conclusion}

Echocardiographic techniques are the initial and complementary cardiac imaging tools in the IE diagnosis and follow-up. They present a sensitivity around $90 \%$ for IE diagnosis and in $85-90 \%$ of cases of IE the diagnosis is based on echocardiographic criteria. A normal TTE does not exclude the IE diagnosis and, in high-risk cases, it is recommended to perform a TEE or repeat another examination after 7-10 days. Nevertheless, it is mandatory to individualized each case and agree with the members of the Endocarditis Team on the course of action to be taken. The echocardiography also provides important information about the patient's prognosis and in positive cases, follow-up echocardiography should be performed. Due to the evolution of technology, echocardiography is increasingly performed by more specialists and its use in the field of IE is rapidly expanding.

\section{Acknowledgment}

The authors are thankful to "Unitat d'Imatge Cardíaca Avançada of Hospital Josep Trueta", for helping us with the echocardiography images and videos used in this review.

\section{Author's Contributions}

Josep Maria Frigola: Coordinated the data-analysis and contributed to the writing of the manuscript.

Sergio Moral: Contributed to writing the original draft, reviewing and editing.

Pau Vilardell and Ramon Brugada: Contributed to the writing and reviewing of the Manuscript.

\section{Ethics}

This article is original and contains unpublished material. The corresponding author confirms that all of the other authors have read and approved the manuscript and there are no ethical issues involved.

\section{References}

Afonso, L., Kottam, A., Reddy, V., \& Penumetcha, A. (2017). Echocardiography in infective endocarditis: state of the art. Current Cardiology Reports, 19(12), 1-13. https://doi.org/10.1007/s11886-017-0928-9

Bhattacharyya, S., Bahrami, T., \& Rahman-Haley, S. (2014). Comprehensive assessment of complications of infective endocarditis by 3D transesophageal echocardiography. International Journal of Cardiology, 174(3), e87-9. https://doi.org/10.1016/j.ijcard.2014.04.029

Bugg, C. W., \& Berona, K. (2016). Point-of-care ultrasound diagnosis of left-sided endocarditis. Western Journal of Emergency Medicine: Integrating Emergency Care with Population Health, 17(3), 383. https://doi.org/10.5811/westjem.2016.2.29921 
Cabell, C. H., Jollis, J. G., Peterson, G. E., Corey, G. R., Anderson, D. J., Sexton, D. J., ... \& Fowler, V. G. (2002). Changing patient characteristics and the effect on mortality in endocarditis. Archives of Internal Medicine, 162(1), 90-94. https://doi.org/10.1001/archinte.162.1.90

Daniel, W. G., Mügge, A., Martin, R. P., Lindert, O., Hausmann, D., Nonnast-Daniel, B., ... \& Lichtlen, P. R. (1991). Improvement in the diagnosis of abscesses associated with endocarditis by transesophageal echocardiography. New England Journal of Medicine, 324(12), 795-800. https://doi.org/10.1056/NEJM199103213241203

de Isla, L. P., Sánchez, S. D., Pagola, J., de Casasola Sánchez, G. G., Fernández, T. L., Barrancos, I. M. S., ... \& Macho, J. T. (2018). Consensus document of the SEMI, semFYC, SEN and SEC on focused cardiac ultrasound in Spain. Revista Española de Cardiología (English Edition), 71(11), 935-940.

https://doi.org/10.1016/j.rec.2018.05.039.

del Prado Díaz, S., Refoyo Salicio, E., Valbuena-Lopez, S. C., Fernández-Velilla Peña, M., Ramírez-Valdiris, U., \& Guzmán-Martínez, G. (2016). Utilidad de la tomografía computarizada con multidetectores en la endocarditis infecciosa. Revista Española de Cardiología, 69(4), 442-445. https://doi.org/10.1016/j.recesp.2015.12.007

Erba, P. A., Pizzi, M. N., Roque, A., Salaun, E., Lancellotti, P., Tornos, P., \& Habib, G. (2019). Multimodality imaging in infective endocarditis: an imaging team within the endocarditis team. Circulation, 140(21), 1753-1765.

https://doi.org/10.1161/CIRCULATIONAHA.119.0 40228

Evangelista, A., \& Gonzalez-Alujas, M. T. (2004). Echocardiography in infective endocarditis. Heart, 90(6), 614-617. https://doi.org/10.1136/hrt.2003.029868.

Fernandez-Hidalgo, N., \& Almirante, B. (2012). Infective endocarditis in the XXI century: epidemiological, therapeutic and prognosis changes. Enfermedades Infecciosas y Microbiologia Clinica, 30(7), 394-406. https://doi.org/10.1016/j.eimc.2011.11.005

Fischer, B. G., \& Baduashvili, A. (2019). Cardiac Pointof-Care Ultrasound for the Diagnosis of Infective Endocarditis in a Patient with Non-Specific Rheumatologic Symptoms and Glomerulonephritis. The American Journal of Case Reports, 20, 542. https://doi.org/10.12659/AJCR.914708

García-Granja, P. E., López, J., Vilacosta, I., Sarriá, C., Domínguez, F., Ladrón, R., ... \& San Román, J. A. (2020). Modelo predictivo de mortalidad hospitalaria en endocarditis infecciosa izquierda. Revista Española de Cardiología, 73(11), 902-909. https://doi.org/10.1016/j.recesp.2020.04.010
Habib, G. (2005). Embolic risk in subacute bacterial endocarditis: determinants and role of transesophageal echocardiography. Current Infectious Disease Reports, 7(4), 264-271. https://doi.org/10.1007/s11908-005-0058-1

Habib, G., Badano, L., Tribouilloy, C., ... \& European Association of Echocardiography. (2010). Recommendations for the practice of echocardiography in infective endocarditis. European Journal of Echocardiography, 11(2), 202-219. https://doi.org/10.1093/ejechocard/jeq004

Habib, G., Erba, P. A., Iung, B., Donal, E., Cosyns, B., ... \& Lancellotti, P. (2019). Clinical presentation, aetiology and outcome of infective endocarditis. Results of the ESC-EORP EURO-ENDO (European infective endocarditis) registry: a prospective cohort study. European Heart Journal, 40(39), 3222-3232. https://doi.org/10.1093/eurheartj/ehz620

Habib, G., Lancellotti, P., Antunes, M. J., Bongiorni, M. G., Casalta, J. P., Del Zotti, F., ... \& Zamorano, J. L. (2015). 2015 ESC guidelines for the management of infective endocarditis: the task force for the management of infective endocarditis of the European Society of Cardiology (ESC) endorsed by: European Association for Cardio-Thoracic Surgery (EACTS), the European Association of Nuclear Medicine (EANM). European Heart Journal, 36(44), 3075-3128. https://doi.org/10.1093/eurheartj/ehv319

Habib, G., Lancellotti, P., Antunes, M. J., Bongiorni, M. G., Casalta, J. P., Del Zotti, F., ... \& Zamorano, J. L. (2015). 2015 ESC guidelines for the management of infective endocarditis: the task force for the management of infective endocarditis of the European Society of Cardiology (ESC) endorsed by: European Association for Cardio-Thoracic Surgery (EACTS), the European Association of Nuclear Medicine (EANM). European Heart Journal, 36(44), 3075-3128. doi:10.1093/eurheartj/ehv319

Heiro, M., Helenius, H., Hurme, S., Savunen, T., Metsärinne, K., Engblom, E., ... \& Kotilainen, P. (2008). Long-term outcome of infective endocarditis: a study on patients surviving over one year after the initial episode treated in a Finnish teaching hospital during 25 years. BMC Infectious Diseases, 8(1), 1-13. https://doi.org/10.1186/1471-2334-8-49

Hilberath, J. N., Oakes, D. A., Shernan, S. K., Bulwer, B. E., D’Ambra, M. N., \& Eltzschig, H. K. (2010). Safety of transesophageal echocardiography. Journal of the American Society of Echocardiography, 23(11), 1115-1127.

https://doi.org/10.1016/j.echo.2010.08.013

Keynan, Y., Singal, R., Kumar, K., Arora, R. C., \& Rubinstein, E. (2013). Infective endocarditis in the intensive care unit. Critical Care Clinics, 29(4), 923-951. https://doi.org/10.1016/j.ccc.2013.06.011 
Khan, M. Z., Munir, M. B., Khan, M. U., Khan, S. U., Benjamin, M. M., \& Balla, S. (2020). Contemporary trends in native valve infective endocarditis in United States (from the national inpatient sample database). The American Journal of Cardiology, 125(11), 1678-1687. https://doi.org/10.1016/j.amjcard.2020.02.035

Li, J. S., Sexton, D. J., Mick, N., Nettles, R., Fowler Jr, V. G., Ryan, T., ... \& Corey, G. R. (2000). Proposed modifications to the Duke criteria for the diagnosis of infective endocarditis. Clinical Infectious Diseases, 30(4), 633-638. https://doi.org/10.1086/313753

Liu, Y. W., Tsai, W. C., Lin, C. C., Hsu, C. H., Li, W. T., Lin, L. J., \& Chen, J. H. (2009). Usefulness of realtime three-dimensional echocardiography for diagnosis of infective endocarditis. Scandinavian Cardiovascular Journal, 43(5), 318-323. https://doi.org/10.1080/14017430902737940

MacKay, E. J., Neuman, M. D., Fleisher, L. A., Patel, P. A., Gutsche, J. T., Augoustides, J. G., ... \& Groeneveld, P. W. (2020). Transesophageal echocardiography, mortality and length of hospitalization after cardiac valve surgery. Journal of the American Society of Echocardiography, 33(6), 756-762. https://doi.org/10.1016/j.echo.2020.01.014

Martín-Dávila, P., Navas, E., Fortún, J., Moya, J. L., Cobo, J., Pintado, V., ... \& Moreno, S. (2005). Analysis of mortality and risk factors associated with native valve endocarditis in drug users: the importance of vegetation size. American Heart Journal, 150(5), 1099-1106.

https://doi.org/10.1016/j.ahj.2005.02.009

Mügge, A., Daniel, W. G., Frank, G., \& Lichtlen, P. R. (1989). Echocardiography in infective endocarditis: reassessment of prognostic implications of vegetation size determined by the transthoracic and the transesophageal approach. Journal of the American College of Cardiology, 14(3), 631-638. https://doi.org/10.1016/0735-1097(89)90104-6

Nishimura, R. A., Otto, C. M., Bonow, R. O., Carabello, B. A., ErwinIII, J. P., ..., \& Thomas, J. D. (2014). 2014 AHA/ACC Guideline for the Management of Patients with Valvular Heart Disease: Executive Summary: A Report of the American College of Cardiology/American Heart Association Task Force on Practice Guidelines. Circulation, 129, 2440-2492. https://doi.org/10.1161/CIR.0000000000000029

Olmos, C., Vilacosta, I., Fernández-Pérez, C., Bernal, J. L., Ferrera, C., García-Arribas, D., ... \& Elola, F. J. (2017). The evolving nature of infective endocarditis in Spain: a population-based study (2003 to 2014). Journal of the American College of Cardiology, 70(22), 2795-2804.

https://doi.org/10.1016/j.jacc.2017.10.005
Ortiz, C., López, J., García, H., Sevilla, T., Revilla, A., Vilacosta, I., ... \& San Román, J. A. (2014). Clinical classification and prognosis of isolated right-sided infective endocarditis. Medicine, 93(27), 1-6. https://doi.org/10.1097/MD.0000000000000137

Ortiz-Bautista, C., López, J., García-Granja, P. E., Sevilla, T., Vilacosta, I., Sarriá, C., ... \& San Román, J. A. (2015). Current profile of infective endocarditis in intravenous drug users: the prognostic relevance of the valves involved. International Journal of Cardiology, 187, 472-474. https://doi.org/10.1016/j.ijcard.2015.03.368

Otto, C. M., Nishimura, R. A., Bonow, R. O., Carabello, B. A., Erwin III, J. P., ..., \& Toly, C. (2020) ACC/AHA guideline for the management of patients with valvular heart disease: A report of the american college of cardiology/American heart association joint committee on clinical practice guidelines. Circulation, 143, e72-e227. https://doi.org/10.1161/CIR.0000000000000923

Pant, S., Patel, N. J., Deshmukh, A., Golwala, H., Patel, N., Badheka, A., ... \& Mehta, J. L. (2015). Trends in infective endocarditis incidence, microbiology and valve replacement in the United States from 2000 to 2011. Journal of the American College of Cardiology, 65(19), 2070-2076. https://doi.org/10.1016/j.jacc.2015.03.518

Revilla, A., López, J., Villacorta, E., Gómez, I., Sevilla, T., del Pozo, M. Á., ... \& San Román, J. A. (2008). Endocarditis derecha aislada en pacientes no adictos a drogas por vía parenteral. Revista Española de Cardiología, 61(12), 1253-1259. https://doi.org/10.1016/S0300-8932(08)75732-4

Seif, D., Meeks, A., Mailhot, T., \& Perera, P. (2013). Emergency department diagnosis of infective endocarditis using bedside emergency ultrasound. Critical Ultrasound Journal, 5(1), 1-4. https://doi.org/10.1186/2036-7902-5-1

Shah, A. S., McAllister, D. A., Gallacher, P., Astengo, F., Rodríguez Pérez, J. A., Hall, J., ... \& Cruden, N. L. (2020). Incidence, Microbiology and Outcomes in Patients Hospitalized With Infective Endocarditis. Circulation, 141(25), 2067-2077. https://doi.org/10.1161/circulationaha.119.044913

Shapira, Y., Weisenberg, D. E., Vaturi, M., Sharoni, E., Raanani, E., Sahar, G., ... \& Sagie, A. (2007). The impact of intraoperative transesophageal echocardiography in infective endocarditis. IMAJ-RAMAT GAN-, 9(4), 299. https://pubmed.ncbi.nlm.nih.gov/17491226/

Sochowski, R. A., \& Chan, K. L. (1993). Implication of negative results on a monoplane transesophageal echocardiographic study in patients with suspected infective endocarditis. Journal of the American College of Cardiology, 21(1), 216-221. https://doi.org/10.1016/0735-1097(93)90739-n 
Taylor, J., Braver, O., \& Fuchs, L. (2017). Infective Endocarditis Diagnosed by Pocket Ultrasound at the Bedside. Journal of ultrasound in medicine: official journal of the American Institute of Ultrasound in Medicine, 36(11), 2392-2394. https://doi.org/10.1002/jum.14299

Thuny, F., Avierinos, J. F., Tribouilloy, C., Giorgi, R., Casalta, J. P., Milandre, L., ... \& Habib, G. (2007). Impact of cerebrovascular complications on mortality and neurologic outcome during infective endocarditis: a prospective multicentre study. European Heart Journal, 28(9), 1155-1161. https://doi.org/10.1093/eurheartj/ehm005

Thuny, F., Disalvo, G., Belliard, O., Avierinos, J. F., Pergola, V., Rosenberg, V., ... \& Habib, G. (2005). Risk of embolism and death in infective endocarditis: prognostic value of echocardiography: a prospective multicenter study. Circulation, 112(1), 69-75. https://doi.org/10.1161/circulationaha.104.493155

Thuny, F., Grisoli, D., Collart, F., Habib, G., \& Raoult, D. (2012). Management of infective endocarditis: challenges and perspectives. The Lancet, 379(9819), 965-975. https://doi.org/10.1016/S0140-6736(11)60755-1

Tornos, P., Gonzalez-Alujas, T., Thuny, F., \& Habib, G. (2011). Infective endocarditis: the European viewpoint. Current Problems in Cardiology, 36(5), 175-222. https://doi.org/10.1016/j.cpcardiol.2011.03.004

Tran, H. M., Truong, V. T., Ngo, T. M., Bui, Q. P., Nguyen, H. C., Le, T. T., ... \& Pham, V. N. (2017). Microbiological profile and risk factors for inhospital mortality of infective endocarditis in tertiary care hospitals of south Vietnam. PLoS One, 12(12), e0189421.

https://doi.org/10.1016/j.jacc.2017.07.564
Vieira, M. L. C., Grinberg, M., Pomerantzeff, P. M. A., Andrade, J. L., \& Mansur, A. J. (2004). Repeated echocardiographic examinations of patients with suspected infective endocarditis. Heart, 90(9), 1020-1024. https://doi.org/10.1136/hrt.2003.025585

Vilacosta, I., Olmos, C., de Agustín, A., López, J., Islas, F., Sarriá, C., ... \& San Román, A. (2015). The diagnostic ability of echocardiography for infective endocarditis and its associated complications. Expert review of cardiovascular therapy, 13(11), 1225-1236. https://doi.org/10.1586/14779072.2015.1096780

Yuan, X. C., Liu, M., Hu, J., Zeng, X., Zhou, A. Y., \& Chen, L. (2019). Diagnosis of infective endocarditis using echocardiography. Medicine, 98(38). https://doi.org/10.1097/MD.0000000000017141
Abbreviations
ED: Emergency Department
ICU: Intensive Care Unit
IE: Infective Endocarditis
POCUS: Point-Of-Care Ultrasound
TEE: Transesophageal Echocardiography
TTE: Transthoracic Echocardiography
CT: Computed Tomography scan) 